\title{
First Experiences in 200 Thrust Plate Prosthesis
}

\section{Sander B Schouten*, Peter J van Winterswijk, Leon W Diederix, Jaap Huij and Pieter A Bakx}

Department of Orthopedics, Maasstad Hospital, Netherlands

${ }^{*}$ Corresponding author: Sander B Schouten, Maasstad Hospital, Secretariat Orthopedics, 3079 DZ Rotterdam, The Netherlands; E-mail: sbschouten@hotmail.com

Received date: January 28, 2016; Accepted date: March 07, 2016; Published date: March 10, 2016

Copyright: (C) 2016, Schouten SB, et al. This is an open-access article distributed under the terms of the Creative Commons Attribution License, which permits unrestricted use, distribution, and reproduction in any medium, provided the original author and source are credited.

\section{Abstract}

Background: The thrust plate prosthesis (TPP) is an alternative to a stemmed total hip prosthesis. This stemless femoral implant designed for total hip arthroplasty (THA) was developed to simulate the physiological loading of the metaphysis of the proximal femur and can be used in young patients.

Methods and findings: This retrospective study describes the mid-term results of a cohort of 171 patients (in total 200 procedures) who received a TPP for the indication of primary osteoarthritis of the hip. These mid-term results are defined by the complication free survival, which includes dislocation, periprosthetic fracture, infection, thromboembolism, pneumonia, and cardiologic complications.

Conclusions: Of the 200 procedures the complication free survival rate was $94 \%$ and the revision free survival rate was $97.5 \%$ with a mean follow-up of 9.03 years.

Keywords: Thrust plate prosthesis; Mid-term results; Total hip arthroplasty

\section{Introduction}

The replacement of a hip joint by a total hip prosthesis is a successful and frequently performed procedure in orthopaedic surgery. In the Netherlands, every year approximately 23.000 total hip arthroplasties are carried out [1].

The Thrust Plate Prosthesis (TPP) (Zimmer, Winterthur, Switzerland) is a total hip prosthesis (Figure 1) which can be used as a preserving bone stock technique. The TPP was introduced by Huggler and Jacob in 1978. This stemless femoral implant designed for total hip arthroplasty (THA) was developed to simulate the physiological loading of the metaphysis of the proximal femur. Results of biomechanical studies of the TPP have proven this simulation to be accurate [2-6]. The proximal fixation of the TPP makes possible revision procedures relatively less complex, as they can now be performed using a stemmed prosthesis normally used for primary THA [7].

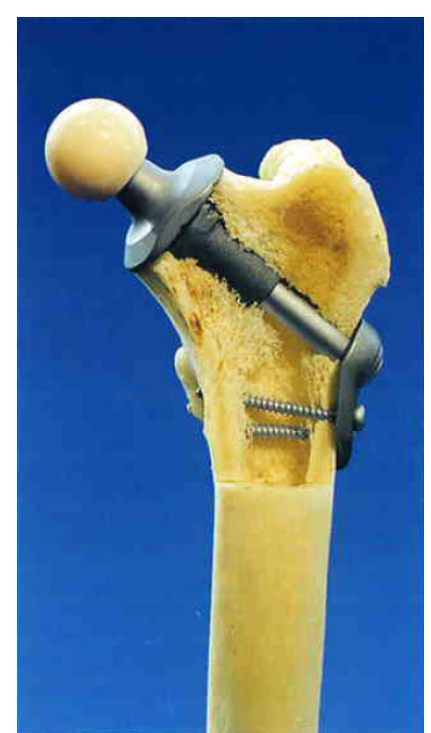

Figure 1: a cross section view of The Thrust Plate Prosthesis in a sawbone.

The aim of this retrospective study was to describe the midterm results of the first 200 TPP's in the Maasstad Hospital.

\section{Methods}

Between July 1997 and March 2007200 TPP's were placed in 171 consecutive patients in the Maasstad Hospital. The third generation TPP which was used, has an oval thrust plate to fit the femoral neck, is made of titaniumalloy (TiAINb) and cobaltchromium-molybdenum (CoCrMo) and the shape of the lateral plate prevents it from moving proximo-laterally and prevents breakage of the screw-arms through which the screws were drilled. There is no hydroxyapatite coating. The texture of the underside of the thrust plate is corundum blasted to enhance bone on growth. The bolt is forged CoCrMo. There are three thrust plate sizes: 44,40 and $38 \mathrm{~mm}$, and the bolt is also produced in three different lengths: 73,81 and $89 \mathrm{~mm}$.

In our study, out of the 171 patients 84 were male and 87 were female. The patients had a mean age of 54.6 years (1981 years) (median $=55.6$ years) at the time of surgery.

The indication for total hip arthroplasty in these young patients was primary osteoarthritis of the hip in 160 patients, 
femoral head necrosis in 10 patients, secondary osteoarthritis to hip dysplasia in 6 patients, posttraumatic osteoarthritis in 9 patients and rheumatoid disease in 15 patients.

All patients were diagnosed, clinically and radiologically, with primary osteoarthritis of the hip. All patients had undergone at least 6 months of conservative therapy including oral analgesics, physiotherapy and weight reduction. Two orthopaedic surgeons (PB and $\mathrm{JH}$ ) performed the operations. In all cases they aimed to create a caput-collum-diaphysis (CCD) angle of 130 degrees for biomechanical reasons. A monoblock cementless Morscher press-fit acetabular component was used (Zimmer, Winterthur, Switzerland). The head diameter was $28 \mathrm{~mm}$ or $32 \mathrm{~mm}$. Until September 2004 a head diameter of $28 \mathrm{~mm}$ was used in 136 TPP's. From September 2004 onward a head diameter of $32 \mathrm{~mm}$ was used in 64 TPP's. In all patients the postoperative management consisted of 6 weeks $10 \%$ partial weight bearing. Results of the acetabular component will not be described in this article.

This study describes the mid-term results of our first 200 consecutive TPP's. These mid-term results are defined by the complication free survival, which includes dislocation, periprosthetic fracture, infection, thromboembolism, pneumonia, and cardiologic complications.

\section{Surgical technique}

The procedures were performed with a straight lateral approach as described in Diederix et al. and illustrated by figures 2-4 [7]: under spinal anaesthesia, the patient was placed in the lateral decubitus position. A straight lateral approach was made. Distal to the greater trochanter, the proximal attachment of the lateral vastus muscle was incised in the direction of the muscle fibers to create sufficient space for the lateral plate of the TPP under the muscle. A $4.5 \mathrm{~mm}$ hole was then drilled through the lateral femoral cortex. Arthrotomy was performed and the hip was dislocated anteriorly. A Kirschner wire and angle-measuring device were used to check for the possibility of having a CCD-angle of $130^{\circ}$. The femoral head was resected after osteotomy of the neck in a plane perpendicular to its axis. With an aiming device, a central hole was drilled from the lateral cortex through the femoral neck. The acetabulum was reamed and the acetabular component was placed. With the leg in external rotation, the size of the thrust plate was determined. The cancellous bone of the femoral neck was then compressed with a rasp. The TPP was introduced through gentle knocking to allow the bone to adapt progressively to compression. The TPP screw was fixed through the lateral plate in the thrust plate. Two cortical screws secured the lateral plate. A ceramic femoral head was placed; the hip was relocated and checked for stability. The wound was then closed. Postoperative management consisted of 6 weeks $10 \%$ weight bearing.
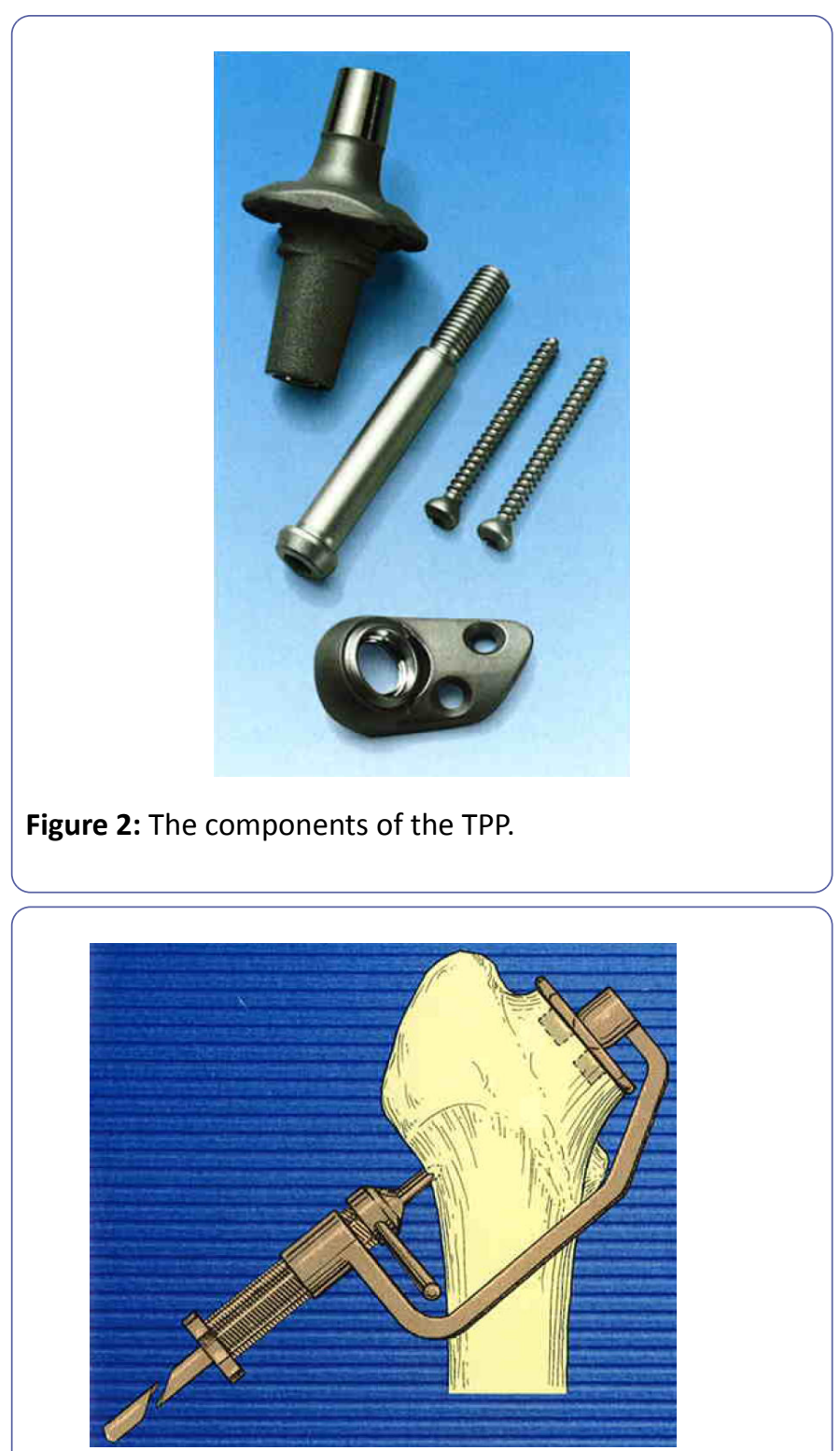

Figure 3: With an aiming device, a central hole is drilled from the lateral cortex through the femoral neck.

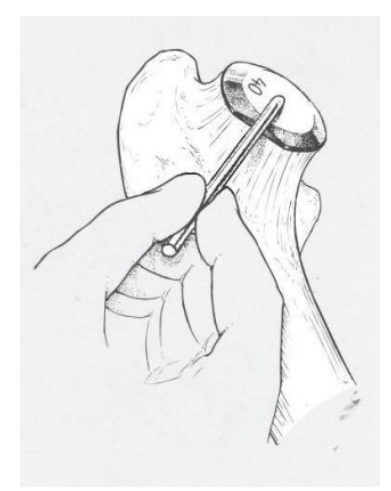

Figure 4: The surface size of the thrust plate is measured. 


\section{Results}

The follow-up period ranged from 2 - 15 years (mean 9.03 years ( \pm 3.05 years)). Two patients died, but the causes of their deaths (oesophageal- and oropharyngeal cancer) were unrelated to the arthroplasty. We performed a retrospective case series analysis and the analysis of the data took place in July 2014.

In 12 out of 200 (6\%) TPP's complications occurred. Five patients $(2.5 \%)$ underwent a revision arthroplasty of the TPP. These patients are discussed below and summarized in table 1. The patients in which complications occured had an average age of 51.6 (36-62 years) at the time of surgery. Five of them were male and seven were female.

Table 1: Five patients which underwent a revision arthroplasty of the TPP.

\begin{tabular}{|c|c|c|c|c|}
\hline Age & Indication & $\begin{array}{l}\mathrm{m} / \\
\mathrm{f}\end{array}$ & $\begin{array}{l}\text { Revision } \\
\text { after }\end{array}$ & treatment \\
\hline 47 & $\begin{array}{l}\text { A radiological } \\
\text { progressive } \\
\text { varisation of } \\
\text { the left TPP }\end{array}$ & $\mathrm{m}$ & 32 months & Revision with a CLS-stem \\
\hline 49 & $\begin{array}{l}\text { A radiological } \\
\text { progressive } \\
\text { varisation of } \\
\text { the left TPP }\end{array}$ & $\mathrm{m}$ & 16 months & Revision with a CLS-stem \\
\hline 42 & $\begin{array}{l}\text { Dislocations / } \\
\text { subluxations }\end{array}$ & $f$ & 91 months & $\begin{array}{l}\text { The acetabular Morscher } \\
\text { cup was replaced by a } \\
\text { RM-cup (Mathys) and a } \\
\text { head diameter of } 28 \mathrm{~mm} \\
\text { was replaced by a head } \\
\text { diameter of } 36 \mathrm{~mm} \text {. }\end{array}$ \\
\hline 54 & $\begin{array}{l}\text { Femoral } \\
\text { fracture just } \\
\text { millimeters } \\
\text { distal to the } \\
\text { lateral plate }\end{array}$ & $f$ & 12 months & $\begin{array}{l}\text { The lateral TPP plate was } \\
\text { replaced by a longer, six- } \\
\text { hole lateral TPP plate to } \\
\text { bridge the fracture without } \\
\text { revision of the TPP itself }\end{array}$ \\
\hline 39 & $\begin{array}{l}\text { Progressive } \\
\text { varisation of } \\
\text { the TPP }\end{array}$ & $\mathrm{m}$ & 177 months & Revision with a CLS-stem \\
\hline
\end{tabular}

In the first patient, the indication for total hip arthroplasty was femoral head necrosis after a femoral neck fracture. His history included alcohol abuse. He was operated in March 1999 at the age of 47 and a TPP was implanted on the left side. Two years later he developed pain in the left hip. Radiological evaluation showed varisation of the left TPP. 32 Months after the primary operation a revision arthroplasty was performed with a CLS-stem (Zimmer), with the suspicion of aseptic loosening. The acetabular component was left in place. After a few years the patient died from an unrelated orthopaedic cause.

The second patient underwent a TPP on the left side in June 2000 at the age of 49. His history included morbus Bechterew and myocardial infarction. In June 2001 a TPP was placed on the right side and in October 2001 a radiological progressive varisation of the left TPP was noticed. Therefore, a revision arthroplasty was performed on the left side using a CLS-stem.

This femoral component also had to be revised due to loosening 41 months after implantation. An undiagnosed lowgrade infection may have been the cause of the early failure of the left TPP and CLS. Before reoperation, the serum C-reactive protein was $76 \mathrm{mg} / \mathrm{l}$. The diagnostic work-up did not include bone scintigraphy nor image guided joint aspiration and culture. Peroperative culture was negative, but the stem revision was performed after administration of pre-operative antibiotics. The revision of the CLS was performed with a larger CLS stem and bone impaction grafting, as shown in figures 5 and 6.7 years after the second revision, this implant continues to function.

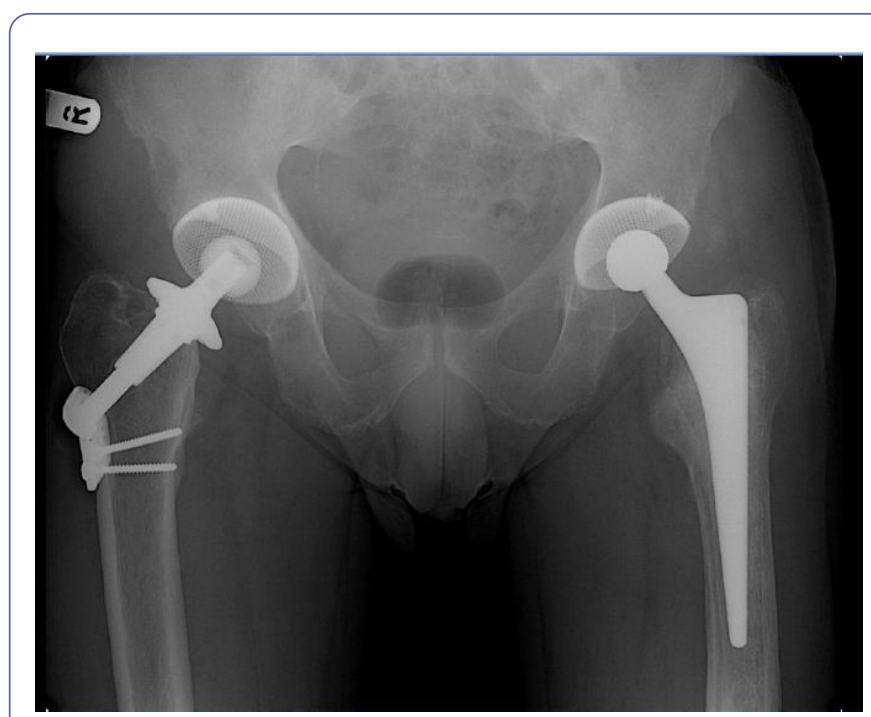

Figure 5: The revision of the CLS was performed with a larger CLS stem and bone impaction grafting.

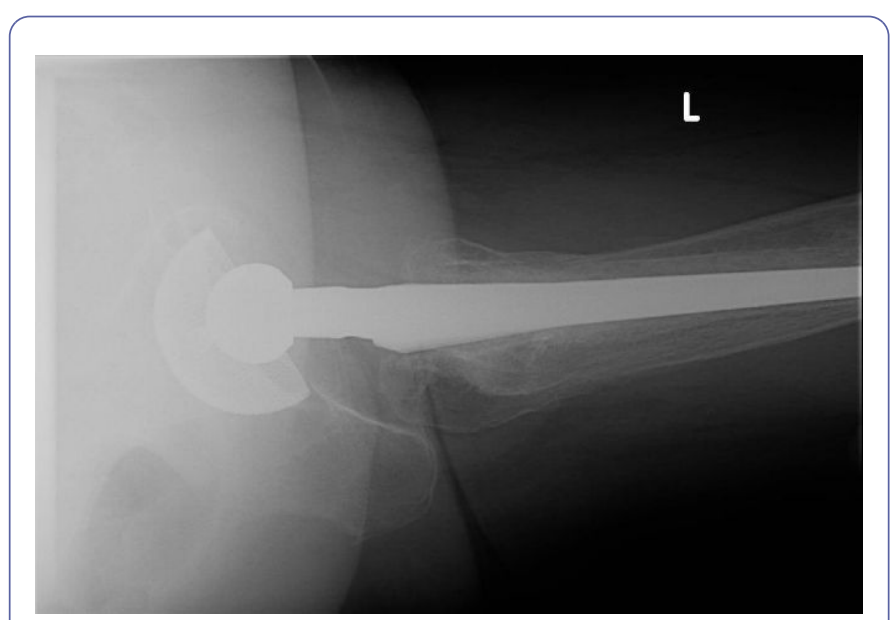

Figure 6: The revision of the CLS was performed with a larger CLS stem and bone impaction grafting.

The third patient was given a TPP on the left side in October 2002 at the age of 42 because of primary osteoarthritis of the 
hip. After 17 months she developed a dislocation twice. Thereafter, several subluxations took place. After being treated with a dislocation brace for several years, in May 2010 (91 months after the primary operation) a revision arthroplasty was performed. The acetabular Morscher cup was replaced by a RM-cup (Mathys) and a head diameter of $28 \mathrm{~mm}$ was replaced by a head diameter of $36 \mathrm{~mm}$, as shown in figures 7 and 8 (preoperatively) and figures 9 and 10 (postoperatively).

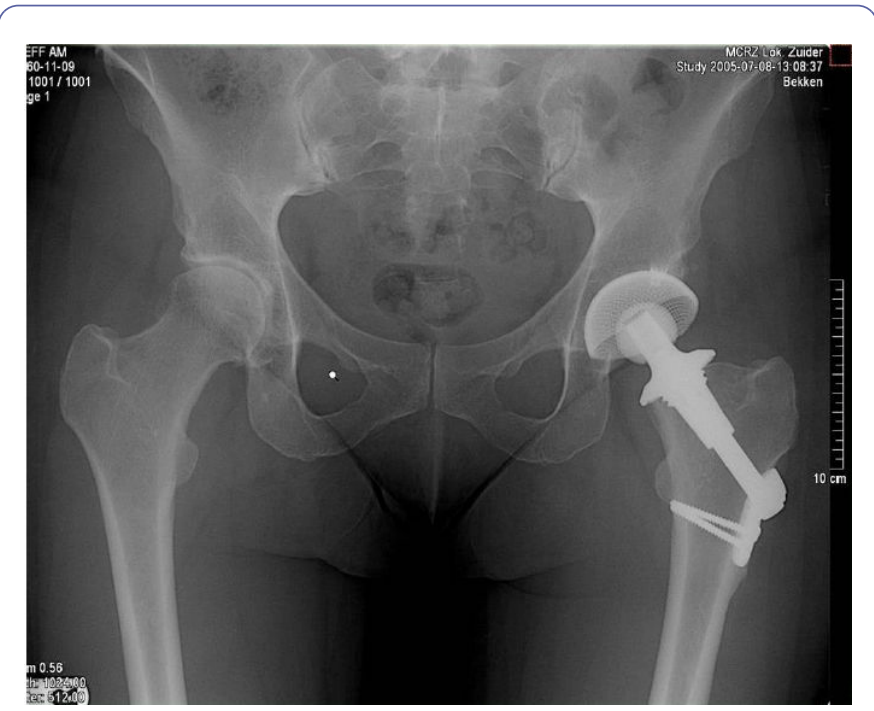

Figure 7: The acetabular Morscher cup was replaced by a RM-cup (Mathys) and a head diameter of $28 \mathrm{~mm}$ was replaced by a head diameter of $36 \mathrm{~mm}$ (preoperatively).

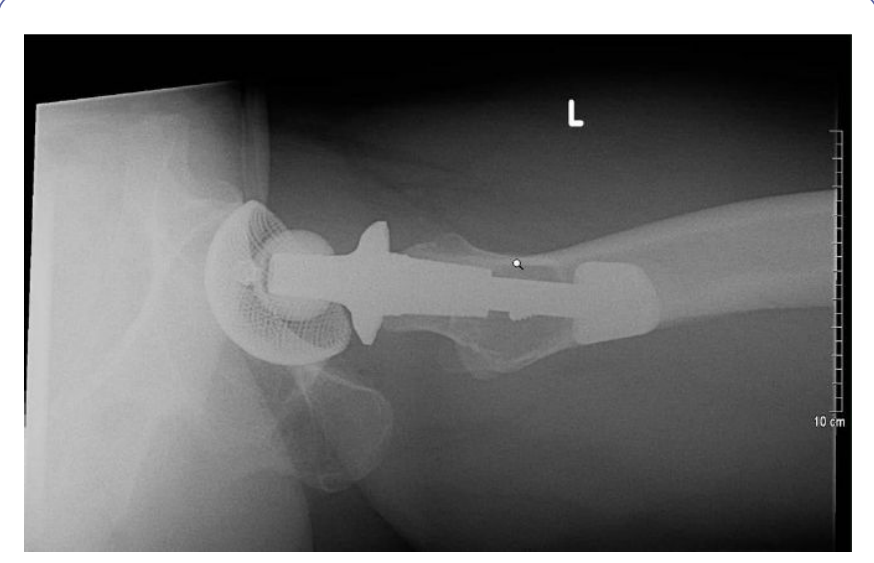

Figure 8: The acetabular Morscher cup was replaced by a RM-cup (Mathys) and a head diameter of $28 \mathrm{~mm}$ was replaced by a head diameter of $36 \mathrm{~mm}$ (preoperatively).

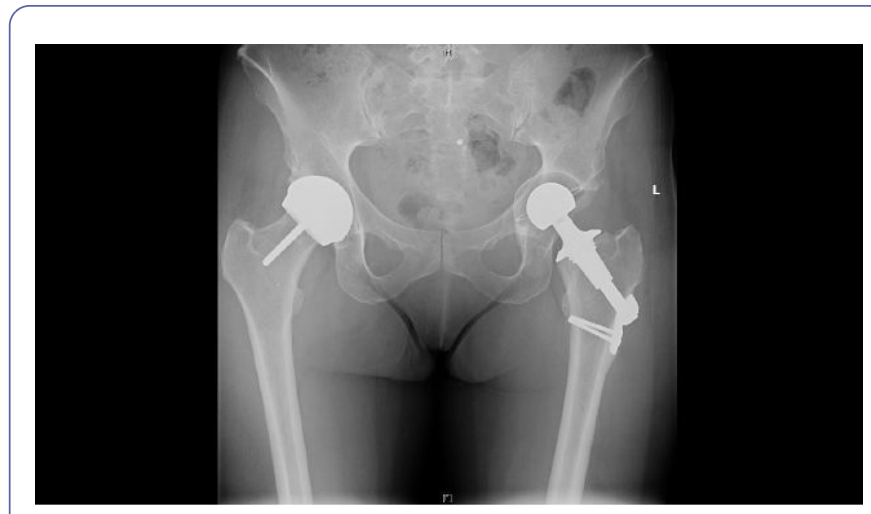

Figure 9: The acetabular Morscher cup was replaced by a RM-cup (Mathys) and a head diameter of $28 \mathrm{~mm}$ was replaced by a head diameter of $36 \mathrm{~mm}$ (postoperatively).

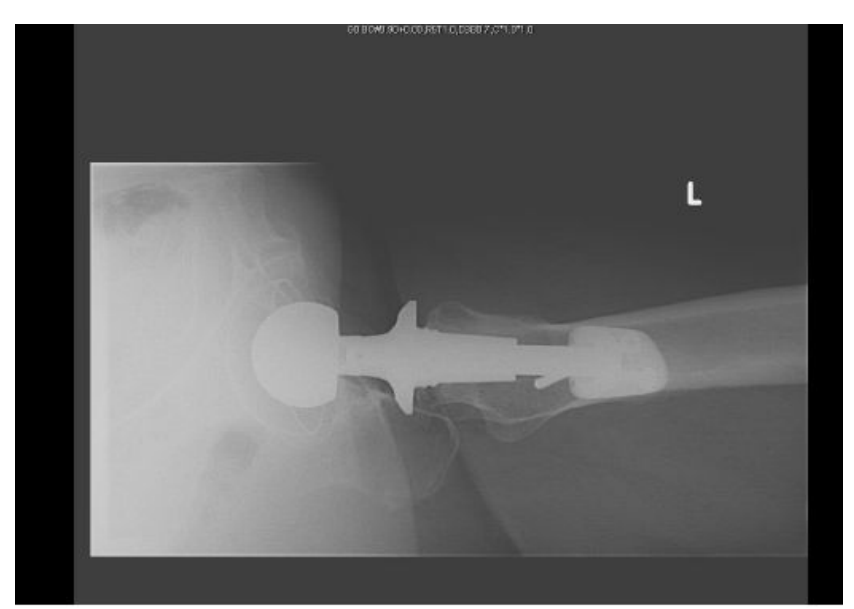

Figure 10: The acetabular Morscher cup was replaced by a RM-cup (Mathys) and a head diameter of $28 \mathrm{~mm}$ was replaced by a head diameter of $36 \mathrm{~mm}$ (postoperatively).

The fourth patient was given a TPP for primary osteoarthritis of the right hip in March 2000 at the age of 54. Six months thereafter, she received a TPP on the contralateral side, because of primary osteoarthritis of the left hip. Six months later, she developed a femoral fracture just millimeters distal to the lateral plate. Therefore, the lateral TPP plate was replaced by a longer, six-hole lateral TPP plate to bridge the fracture without revision of the TPP itself, as shown in figure 11. This type of fracture has been treated and described earlier [7]. She recovered without further complications. 


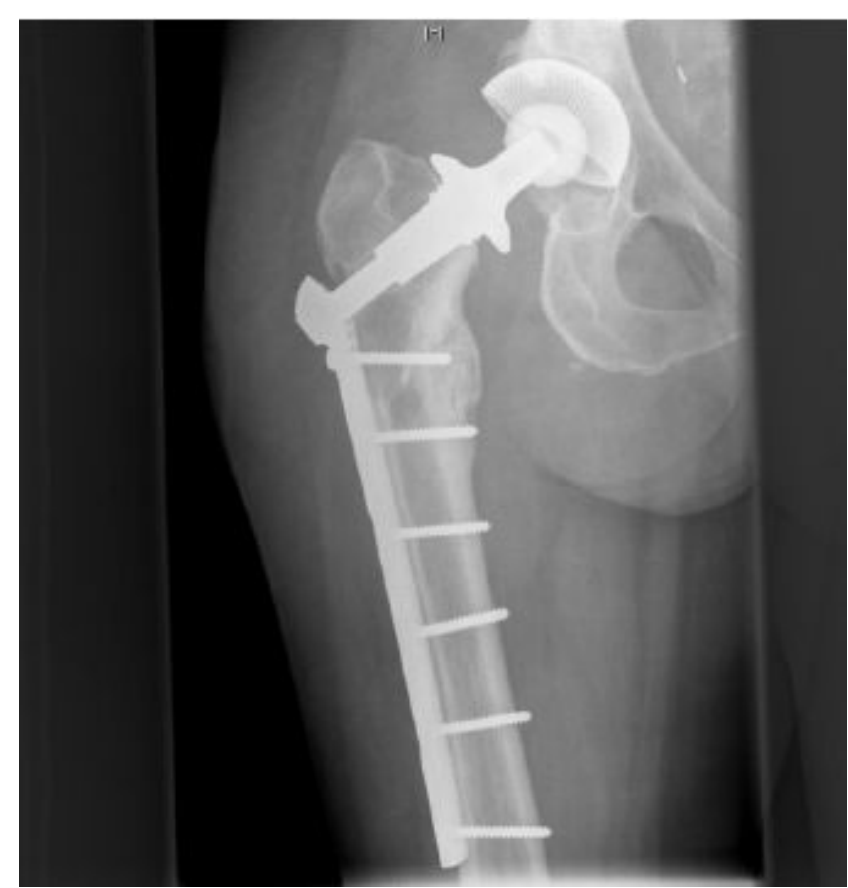

Figure 11: the lateral TPP plate was replaced by a longer, sixhole lateral TPP plate to bridge the fracture without revision of the TPP itself.

The fifth patient who underwent a reoperation, received a TPP at the age of 39 . He had primarily undergone open reduction and internal fixation of an acetabular fracture with resection of the femoral head. He developed slow progressive varisation of the TPP, which started 25 months postoperatively, as shown in figures 12 and 13. After being asymptomatic (with a HHS of 84.2) for several years, he developed progressive pain 14.8 years postoperatively, and therefore underwent a revision procedure of the TPP, with the suspicion of aseptic loosening.

Four patients (2\%) showed a deep wound infection. These patients had an age ranging from 55 to 62 years old. In all patients at least one debridement and implantation with gentamicin loaded beads was performed. In all four cases it was unnecessary to perform a revision arthroplasty after an average of 80 months. In three patients (1.5\%) a dislocation of the TPP occurred. One patient was reoperated as described earlier. In the other two patients dislocation only occurred once and it was treated conservatively. One patient had a postoperative deep vein thrombosis in the left lower leg (0.5\%). Postoperative complications occurred in $6 \%$ of 200 procedures. The revision-free survival rate is $97.5 \%$ after a mean of 9.03 years.

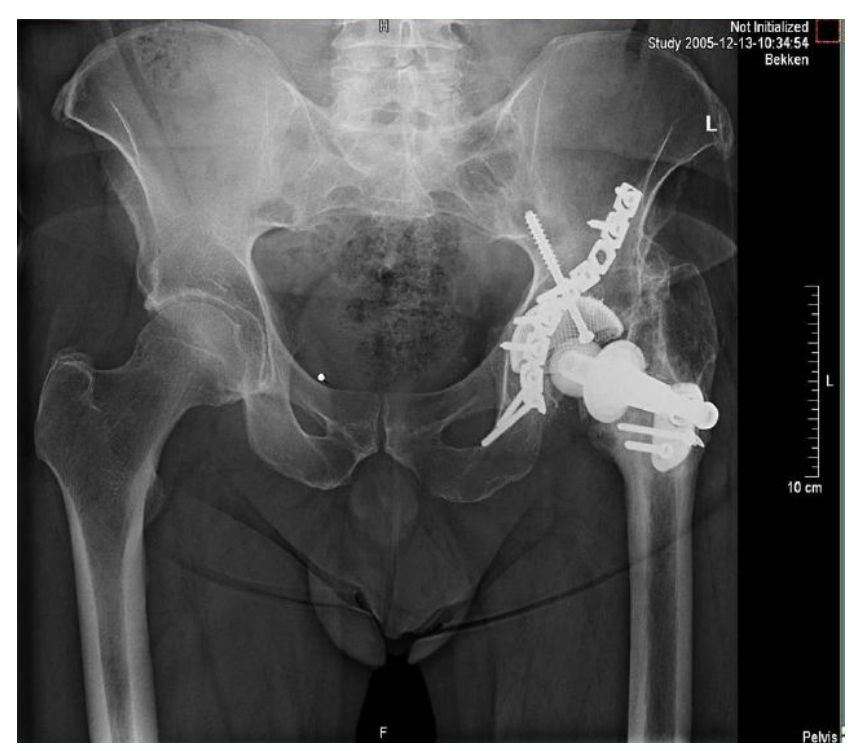

Figure 12: slow progressive varisation of the TPP (postoperatively).

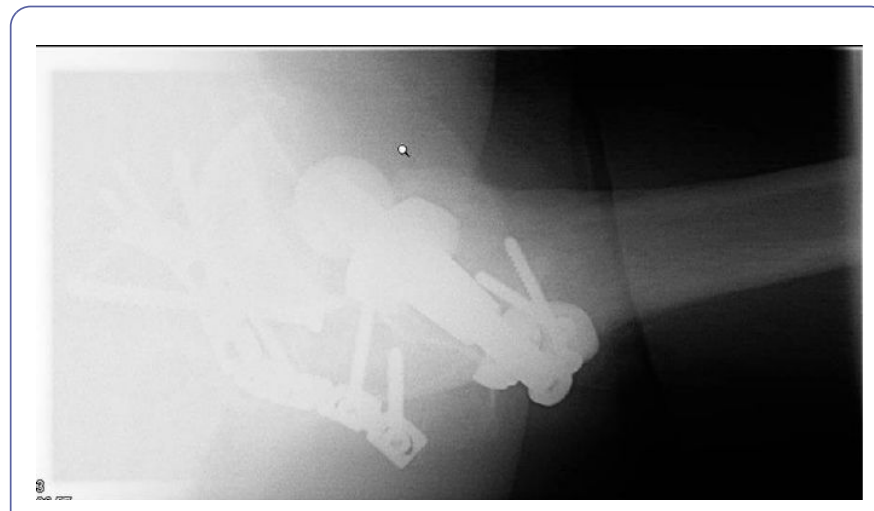

Figure 13: slow progressive varisation of the TPP (postoperatively).

\section{Discussion}

This is one of the largest studies describing the mid-term results of 200 TPP's. The complication free survival rate of $94 \%$ and the revision free survival rate of $97.5 \%$, are both equal to results of other studies concerning the TPP [8-14]. Physiological loading of the proximal femur and preservation of the intramedullary channel are important in THA, especially in young patients. This can be achieved with the TPP. The patients in this retrospective case series analysis had a mean age of 54.6 years ( $19-81$ years) (median $=55.6$ years) at the time of surgery. Only $43.5 \%$ of these patients had an age of lower than 55 years.

The reoperation-free survival was $97.5 \%$ with a mean follow-up of 9.03 years in this series, which complies with the criteria of the National Institute for Health and Clinical Excellence [15]. The learning curve of the two surgeons involved should also be taken into account. The Swedish Hip 
Arthroplasty Register of 2010 showed a reoperation-free survival of approximately $94 \%$ after 12 years for conventional THA's [13]. The Norwegian Arthroplasty Register 2010 showed a reoperation-free survival of approximately $87 \%$ after 12 years [14]. When we extrapolate our data to the criteria of the NICE, this is a favourable result, considering that our population was relatively young and active.

Another alternative for a conventional hip prothesis which is widely used and contains a preserving bone stock technique is the resurfacing hip arthroplasty. The last couple of years hip resurfacing prosthesis have gained increasing popularity for younger, higher-demand patients with degenerative hip pathologies. A recent meta-analysis of Smith et al. [13] shows that patients with Birmingham Hip Resurfacing (HRS), Durom hip resurfacing system and Conserve Plus HRS has better functional outcome and less postoperative luxations, but there were statistically significantly greater incidences of heterotopic ossification $(\mathrm{RR}=1.6, \mathrm{Cl}: 1.2,2.1 ; \mathrm{p}=$ $0.006)$, aseptic loosening ( $R R=3.1 ; 95 \% \mathrm{Cl}: 1.1,8.5 ; \mathrm{p}=0.03)$, and revision surgery $(\mathrm{RR}=1.7, \mathrm{Cl}: 1.2,2.5 ; \mathrm{p}=0.003)$ with HRS compared to total hip arthroplasty [16].

This higher risk of complications has not been demonstrated in TPP's. The TPP may therefore be advantageous in young patients. In the patients where a complication occurred the TPP was easily removed and revised for a CLS stem. Compared to other minimally invasive implants, the indication for the TPP is possibly larger. For example in patients with rheumatoid arthritis, because the fixation seems better than other minimally invasive implants.

Our first experiences in the TPP are positive. In our hospital, patients up to 65 years of age now receive the TPP. This study, in which we describe our first experiences in 200 TPP's, has its limitations. We have no control group, it is a retrospective study and a potential bias lies in the fact that the surgeons who performed the procedures themselves carried out the analysis. This study describes the mid-term results of the TPP. Hopefully we will be able to present the long-term results in the future to analyze whether the revision-free survival rate and complication-free survival rate are comparable with longterm results of conventional total hip prostheses.

There is still a lack of alternative treatments for young patients with osteoarthritis of the hip joint. In conclusion, the TPP could be considered as an alternative to stemmed total hip prosthesis for young patients with primary osteoarthritis of the hip. In 200 TPP's the complication free survival rate was $94 \%$ and the revision free survival rate was $97.5 \%$ with a mean follow-up of 9.03 years. Our findings are comparable with other studies of the TPP. In a revision procedure of the TPP a stem, which would normally be used for a primary THA [17], can be used.

\section{References}

1. Schreurs BW, Busch VJ, Veth RP (2007) Choice of hip prosthesis in patients younger than 50 years. Ned Tijdschr Geneeskd 151: 1918-1922.
2. Bereiter H, Burgi M, Schenk $R$ (1997) Finite element investigations of the proximal femur after implantation of the thrust plate prosthesis compared with findings in a post-mortem histological specimen and in radiological follow-up examinations. In: Huggler $\mathrm{AH}$, Jacob $\mathrm{HA}$, editors. The thrust plate hip prosthesis. Berlin: Springer 48.

3. Fottner A, Schmid M, Birkenmaier C, Mazoochian F, Plitz W, et al. (2009) Biomechanical evaluation of two types of short-stemmed hip prostheses compared to the trust plate prosthesis by threedimensional measurement of micromotions. Clin Biomech 24: 429-434.

4. Goto T, Yasunaga Y, Takahashi K, Ochi M (2004) Biomechanical analysis and quantitative analysis of bone scintigraphy on thrust plate hip prosthesis. Arch Orthop Trauma Surg 124:3 57-362.

5. Jacob HA (2009) Biomechanical principles and design details of the thrust plate prosthesis. In: Huggler AH, Jacob HA, editors. The thrust plate hip prosthesis.

6. Karatosun V, Unver B, Gultekin A, Gunal I (2011) A biomechanical comparison of the thrust plate prosthesis and a stemmed prosthesis. Hip Int 21: 565-570.

7. Diederix LW, Van Winterswijk PJ, Schouten SB, Bakx PA, Huij J (2013) The Thrust Plate Prosthesis: Long-term clinical and radiological results. Acta Orthop Belg 79: 293-300.

8. Buergi ML, Stoffel KK, Jacob HA, Bereiter HH (2005) Radiological findings and clinical results of 102 thrust-plate femoral hip prostheses: a follow-up of 2 to 8 years. J Arthroplasty 20: 108-117.

9. Fink B, Siegmuller C, Schneider T, Conrad S, Schmielau G, et al. (2000) Short- and medium-term results of the thrust plate prosthesis in patients with polyarthritis. Arch Orthop Trauma Surg 120: 294-298.

10. Fink B, Wessel S, Deuretzbacher G, Protzen M, Ruther W (2007) Midterm results of "thrust plate" prosthesis. J Arthroplasty 22: 703-710.

11. Ishaque BA, Gils J, Wienbeck S, Donle E, Basad E, et al. (2009) Eight-year results of the femoral neck prosthesis ESKA-CUT]. Z Orthop Unfall 147: 158-165.

12. Yasunaga $\mathrm{Y}$, Yamasaki T, Matsuo $\mathrm{T}$, Yoshida $\mathrm{T}$, Oshima $\mathrm{S}$, et al. (2012) Clinical and radiographical results of 179 thrust plate hip prostheses: 5-14 years follow-up study. Arch Orthop Trauma Surg 132: 547-554.

13. Garellick G, Karrholm J, Rogmark C, Swedish HP (2010)Hip Arthroplasty Register annual report

14. Nasjonalt (2010)Register for Leddproteser. The Norwegian Arthroplasty Register.

15. Smith TO, Nichols R, Donell ST, Hing CB (2010) The clinical and radiological outcomes of hip resurfacing versus total hiparthroplasty: a meta-analysis and systematic review. Acta Orthop 81: 684-95.

16. Lankester BJ, Smith LK, Spencer RF (2005) Total hip replacement and NICE: new guidelines need to address several areas of uncertainty. BMJ 330: 318-319.

17. Ishaque BA, Gils J, Wienbeck S, Donle E, Basad, et al. (2009) Results after replacement of femoral neck prostheses - thrust plate prosthesis (TPP) versus ESKA cut prosthesis. Z Orthop Unfall 147: 79-88. 